\title{
COMPONENTES ALIMENTICIOS, ESTADO DE ÁNIMO Y SU RELACIÓN CON EL SISTEMA INMUNE EN COVID-19
}

\author{
FOOD COMPONENTS, MOOD AND THEIR RELATIONSHIP WITH THE IMMUNE SYSTEM IN COVID-19. \\ Interián-Gómez Leticia*, Aguila-Gutiérrez Sara Elena*, Esquivias-López Katia Marisol*, Pulido-De la Cruz \\ Victoria Alejandra*, Silva-Arzola Nestor Joshue* González-Becerra Karina*.
}

*Universidad de Guadalajara, Guadalajara, México.

\begin{abstract}
RESUMEN
Introducción: El SARS-CoV-2 causante del Covid-19 no solo afecta al sistema inmunológico, sino también el confinamiento tiene un gran impacto sobre la salud mental y fisiológica de la población. Objetivo: Describir los componentes alimenticios que mejoran el estado de ánimo y su influencia en el fortalecimiento del sistema inmune de la población, durante la pandemia por Covid-19. Material y Método: Se consultaron las bases de datos como Google Scholar y PubMed (MEDLINE), y se incluyeron artículos originales y de revisión. Resultados: Los componentes bioactivos presentes en los alimentos como probióticos, triptófano, L-teanina y antioxidantes, mejoran el estado de ánimo (tristeza, ira, angustia), incluso ayudan a reducir niveles de estrés, ansiedad y depresión; a su vez los probióticos, L-teanina, vitamina $C$ y vitaminas liposolubles ( $A$, D y E) muestran un impacto positivo en el sistema inmunológico. Conclusiones: De acuerdo con la presente revisión, los principales compuestos que se destacan por promover un estado de calma y relajación son el triptófano, la L-teanina y polifenoles encontrados en arándanos y uva morada clase Concord, entre otros alimentos. Probióticos de los géneros Lactobacillus y Bifidobacterium mejoran los estados de ansiedad y depresión. Por su parte, ha sido estudiado el efecto en la respuesta inmune de las vitaminas A, D, E y C, así como de probióticos del género Lactobacillus. Por último, los probióticos y L-teanina destacan por mejorar el estado de ánimo, así como la respuesta inmune.
\end{abstract}

Palabras Clave: Covid-19, compuestos bioactivos, estado de ánimo, sistema inmune.

\section{ABSTRACT}

Introduction: The SARS-CoV-2 that causes Covid-19 not only affects the immune system, but also confinement has a great impact on the mental and physiological health of the population. Objective: To describe bioactive compounds of food that improves mood and their influence on strengthening the immune system of the population, during the Covid-19 pandemic. Material and method: Databases such as Google Scholar and PubMed (MEDLINE) were consulted, and original and review articles were included. Results: The bioactive components present in food such as probiotics, tryptophan, L-Theanine improve mood (sadness, anger, anguish), even can reduce levels of stress, anxiety and depression. Probiotics, L-Theanine, antioxidants such as vitamin C, tryptophan and fat-soluble vitamins ( $A, D$ and $E$ ) showed a positive impact on the immune system. Conclusion: According to our review, the main compounds in promoting a calm and relax mood are tryptophan, $\mathrm{L}$ Theanine and polyphenols found in Blueberries and Concord class purple grapes among others. Lactobacillus probiotics and Bifidobacterium probiotics improve anxiety and depression. Also, it has been studied the effect in the immune response of vitamins A, D, E and C, such as Lactobacillus probiotics. Lastly, probiotics and L-Theanine stand out in improving mood and immune response.

Key words: Covid-19; bioactive compounds, mood, immune system.

Correspondencia: Karina González-Becerra kariglezb@gmail.com

Recibido: 22 de abril 2021, aceptado: 10 de septiembre 2021

(C) Autor2021

(cc) (i)

DOI: https://doi.org/10.29105/respyn20.4-7

Citation: Interián-Gómez L., Aguila-Gutiérrez S.E., Esquivias-López K.M., Pulido-De la Cruz V.A., SilvaArzola N.J. González-Becerra K. (2021) Componentes alimenticios, estado de ánimo y su relación con el sistema inmune en covid-19. Revista Salud Pública y Nutrición, 20 (4), 73-86. 


\section{Artículo de Revisión}

\section{Introducción}

El COVID-19 es la enfermedad transmitida por un nuevo tipo de coronavirus SARS-CoV-2 caracterizada por presentar síntomas leves del resfriado común (fiebre, tos seca, dolor de cabeza, dolor muscular, etc.) y resfriado severo (dificultad al respirar) que desencadenan la muerte (Butlera y Barrientos, 2020; Shakoor et al., 2021). La forma de actuar del virus SARS-CoV-2 es infectar las células humanas por la unión de sus proteínas de pico (S) a la enzima angiotensina-2 (ACE2). La ACE2 se expresa en las células pulmonares AT2, células epiteliales del esófago y en los enterocitos del íleon y el colon (Infusino et al., 2020). La investigación de esta enfermedad ha permitido crear una vacuna con un alto grado de efectividad, pero aún hay retos que alcanzar (Wibawa, T. 2020).

En todo el mundo, el confinamiento por la pandemia de COVID-19 ha tenido un gran impacto en la salud mental de la población, generando tensión emocional que contribuye a presentar ansiedad, estrés y depresión (Speth et al., 2020; Van-Rheenen et al., 2020; Dubey et al., 2020). Tal parece que el estado de ánimo tiene una influencia negativa sobre el sistema inmunológico, confiriendo mayor vulnerabilidad a desarrollar enfermedades infecciosas; desencadenando cambios fisiológicos en el sistema nervioso central, órganos y tejidos periféricos. Por lo que se propone que las emociones negativas pueden tener un impacto nocivo para la salud, una propuesta es a través del debilitamiento del sistema inmunológico y el sistema endocrino (Camacho Arroyo, I. 2020). En este sentido se ha demostrado que existen compuestos bioactivos presentes en los alimentos que pueden alterar las emociones y/o tener influencia sobre el sistema inmunológico. Entre ellos se encuentran los probióticos, el triptófano, la L-teanina y los antioxidantes que mejoran el estado de ánimo. A su vez algunos de éstos muestran también efecto positivo en el sistema inmunológico como los probióticos, el triptófano y la L-teanina; así mismo se ha demostrado la efectividad de las vitaminas A, D, $\mathrm{C}$ y E en el reforzamiento del sistema inmune. Por lo anterior en una dieta saludable debe incluir alimentos ricos en los componentes antes mencionados para tener efectos antiinflamatorios e inmunoreguladores, los cuales modifican el sistema adaptativo que elimina las amenazas de patógenos (Butlera y Barrientos, 2020; Goncalves-Mendes et al., 2019;
Szodoray, et al., 2010) mejorando el bienestar físico y mental.

Por lo tanto, el objetivo de la presente investigación fue describir los componentes alimenticios que mejoran el estado de ánimo y su influencia en el fortalecimiento del sistema inmune de la población, durante la pandemia por Covid-19.

\section{Material y Método}

Se realizó la búsqueda de artículos científicos en el periodo de septiembre del 2020 al mes de abril del 2021 en buscadores como Google Scholar, PubMed, SciELO y Dialnet, utilizando las palabras clave: COVID-19, SARS-CoV-2, nutrition and COVID-19, immune system in COVID-19, anxiety and COVID19, mood and immune system, components and mood, nutrients and immune system, vitamins and mood, tryptophan and mood, probiotics on mood and immune system. Las editoriales consultadas fueron Elsevier, Springer, Wiley, Cambridge University Press, MDPI entre otras.

La selección de artículos se realizó con base en los siguientes criterios: a) artículos publicados del 2010 al 2020, b) artículos originales y de revisión escritos en inglés, c) estudios directamente relacionados con el objetivo de búsqueda y d) estudios realizados en personas adultas. Dentro de las limitaciones del estudio se obtuvo que la información de los artículos se actualiza constantemente con respecto al covid-19, se detectó un déficit de estudios sobre covid-19 y su relación con componentes alimenticios, sistema inmunológico y estado de ánimo durante la búsqueda y revisión de los estudios científicos.

De acuerdo con la Real Academia española las definiciones de términos de importancia en el presente artículo se describen a continuación: Ansiedad: estado de inquietud o zozobra del estado de ánimo. Estrés: tensión provocada por situaciones agobiantes que originan reacciones psicosomáticas o trastornos psicológicos que van de leves a severos. Depresión: síndrome caracterizado por una tristeza profunda y por la inhibición de las funciones psíquicas, a veces con trastornos neurovegetativos.

Pandemia: enfermedad epidémica que se extiende a muchos países o que ataca a casi todos los individuos de una localidad o región. Los compuestos bioactivos se definen como los componentes de los alimentos 
que influyen en las actividades celulares y fisiológicas obteniendo, tras su ingesta, un efecto beneficioso para la salud. Sistema inmune: respuesta o reacción que ocurre dentro de un organismo con el propósito de defenderse de invasores extraños.

\section{Resultados}

Se partió de 1,442 artículos que contenían las palabras clave (mencionadas en el apartado de metodología) en el título o abstract de los artículos. En el primer filtro se excluyeron aquellos que en el título o resumen especificaran que fueron realizados en animales, estudios en otros grupos de edad (niños, adolescentes o ancianos) y componentes dirigidos a mejorar otras condiciones fisiológicas como la memoria y/o sueño, así como alimentos asociados con la sensación de bienestar por su alto contenido en azúcares. Posterior a este filtro se seleccionaron 91 artículos los cuales fueron analizados completamente y de estos se eliminaron 53 por no tener resultados que aportaran a cumplir con el objetivo de la presente revisión es decir que no se demostraba el efecto del componente bioactivo sobre el estado de ánimo y/o el fortalecimiento del sistema inmunológico.

Figura 1. Flujograma de búsqueda y selección de estudios

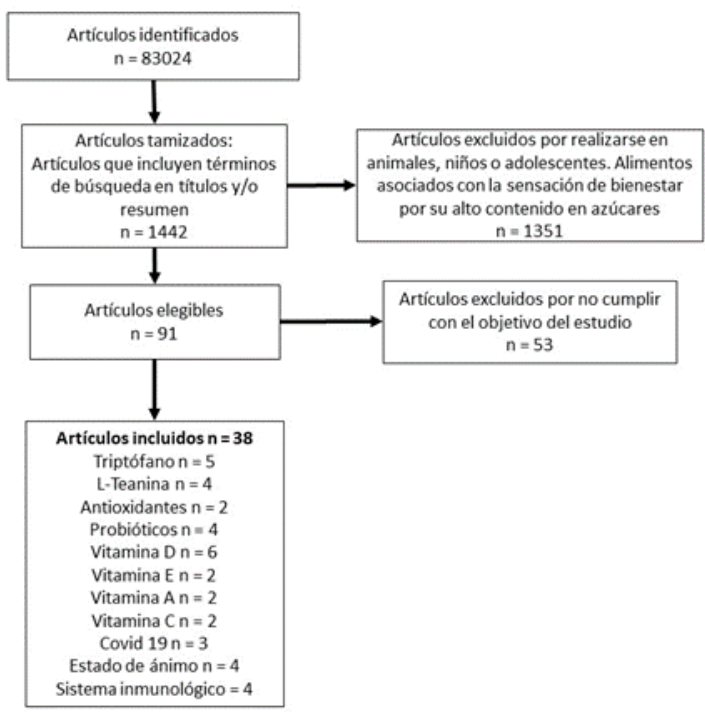

Finalmente, se incluyeron 38 artículos a la sección de desarrollo y discusión de los cuáles 11 artículos fueron sobre antecedentes de covid-19 estado de ánimo y sistema inmunológico, 27 sobre compuestos bioactivos de los cuales 15 mostraron información estadísticamente significativa acerca del efecto de algún compuesto bioactivo sobre las emociones y sistema inmune; con los que se construyeron la Tabla 1 que describe los efectos de componentes alimenticios sobre el estado de ánimo y la tabla 2 que describe los efectos de compuestos alimenticios sobre el sistema inmune. Las tablas se realizaron considerando los siguientes aspectos: alimento, componente alimentario, efecto observado en algún estado de ánimo y/o el sistema inmune y dosis propuesta a la que se obtiene el beneficio descrito.

Como limitaciones de los resultados fue que no se evaluó el riesgo de sesgo de los artículos incluidos en la presente revisión sin embargo todos fueron artículos publicados en revistas indexadas en el Journal Citation Reports de Clarivate Analytics con factor de impacto mayor a 1 lo que sugiere una calidad adecuada de los artículos incluidos en la presente revisión. 
Tabla 1. Efectos de componentes alimenticios en estado de ánimo.

\begin{tabular}{|c|c|c|c|c|c|}
\hline Fuente & Compuesto & Tipo de ensayo & Dosis & Efecto observado & $\begin{array}{c}\text { Alimentos en los que } \\
\text { encuentran }\end{array}$ \\
\hline $\begin{array}{l}\text { (Akkasheh et al., } \\
\text { 2016) }\end{array}$ & $\begin{array}{c}\text { Probióticos } \\
\text { Lactobacillus } \\
\text { acidophilus, } \\
\text { Lactobacillus casei, } \\
\text { Bifidobacterium } \\
\text { bifidum }\end{array}$ & \multirow{2}{*}{$\begin{array}{l}\text { Ensayo clínico } \\
\text { aleatorizado, doble } \\
\text { ciego, } 20 \text { participantes } \\
\text { control recibieron un } \\
\text { placebo y } 20 \\
\text { participantes } \\
\text { suplemento probiótico } \\
\text { en adultos sanos. }\end{array}$} & $\begin{array}{l}3 \mathrm{~g} \text { liofilizado, } \\
\text { probióticos } \\
\text { (OmniBiotic) }\end{array}$ & $\begin{array}{l}\text { Los pacientes que recibieron } \\
\text { suplementos probióticos } \\
\text { disminuyeron } \\
\text { significativamente las } \\
\text { puntuaciones totales del } \\
\text { Inventario de Depresión de } \\
\text { Beck. }\end{array}$ & \multirow{2}{*}{$\begin{array}{l}\text { Bebidas lácteas con } \\
\text { probióticos, yogur, } \\
\text { chucrut, kombucha, } \\
\text { suplementos }\end{array}$} \\
\hline $\begin{array}{l}\text { (Steenbergen et } \\
\text { al., } 2015\end{array}$ & $\begin{array}{l}\text { Probióticos de múlti } \\
\text { especie de } \\
\text { Bifidobacterium }\end{array}$ & & $\begin{array}{l}\text { 2g de polvo } \\
\text { liofilizado, } \\
\text { probióticos } \\
\text { Ecologic Barrier } \\
\text { (probióticos de } \\
\text { Winclove) } \\
\end{array}$ & $\begin{array}{l}\text { Se mostró una reducción en } \\
\text { la depresión, así como la } \\
\text { reducción de la rumia y los } \\
\text { pensamientos agresivos. }\end{array}$ & \\
\hline $\begin{array}{l}\text { (Khalid et al., } \\
\text { 2017) }\end{array}$ & Flavonoides & $\begin{array}{l}\text { Ensayo experimental } \\
\text { aleatorizado, placebo } \\
\text { doble ciego en } 21 \\
\text { adultos jóvenes sanos. }\end{array}$ & $\begin{array}{l}\text { Dosis de una } \\
\text { bebida de } \\
\text { arándanos que } \\
\text { contiene } 253 \mathrm{mg} \\
\text { de antocianina }\end{array}$ & $\begin{array}{l}\text { Menor riesgo de desarrollar } \\
\text { depresión; menor deterioro } \\
\text { de procesos cognitivos que } \\
\text { mantienen la depresión y el } \\
\text { estado de ánimo estable. }\end{array}$ & $\begin{array}{l}\text { Arándanos, moras, } \\
\text { fresas, zarzamoras, } \\
\text { frutas cítricas, } \\
\text { chocolate, nueces, } \\
\text { bebidas derivadas de la } \\
\text { uva como el vino tinto, } \\
\text { té verde y negro. }\end{array}$ \\
\hline $\begin{array}{l}\text { (Haskell-Ramsay, } \\
\text { Stuart, Okello y } \\
\text { Watson, 2017) }\end{array}$ & Antioxidantes & $\begin{array}{l}\text { Ensayo experimental } \\
\text { aleatorizado, doble } \\
\text { ciego, en } 20 \text { adultos } \\
\text { jóvenes sanos. }\end{array}$ & $\begin{array}{l}200 \mathrm{~mL} \text { de jugo } \\
\text { de uva morada } \\
\text { Welch's }+30 \mathrm{~mL} \\
\text { de cordial sabor a } \\
\text { grosella negra } \\
\text { Schweppes } \\
\end{array}$ & $\begin{array}{c}\text { El jugo de uva mejora el } \\
\text { estado de ánimo en adultos } \\
\text { jóvenes sanos, sintiéndose } \\
\text { más tranquilos después de la } \\
\text { uva morada en comparación } \\
\text { con el placebo. }\end{array}$ & Fresas, Moras, Uvas \\
\hline $\begin{array}{l}\text { (Yoto, Motoki, } \\
\text { Murao y } \\
\text { Yokogoshi, 2012) }\end{array}$ & L-teanina & $\begin{array}{l}\text { Ensayo experimental } \\
\text { cruzado, aleatorizado, y } \\
\text { controlado en } 14 \\
\text { personas sanas. }\end{array}$ & $\begin{array}{l}200 \text { mg con } 250 \\
\text { mL de agua }\end{array}$ & Efecto antiestrés & Hojas de Té verde \\
\hline $\begin{array}{l}\text { (Lindseth, Helland } \\
\text { y Caspers, 2015) }\end{array}$ & Triptófano & $\begin{array}{c}\text { Ensayo cruzado } \\
\text { aleatorizado, en } 25 \\
\text { personas jóvenes sanas. }\end{array}$ & $\begin{array}{c}10 \mathrm{mg} / \mathrm{kg} \text { de peso } \\
\text { corporal/día }\end{array}$ & $\begin{array}{l}\text { Disminución de ansiedad y } \\
\text { mejoría en el estado de } \\
\text { ánimo. }\end{array}$ & $\begin{array}{l}\text { Suplementación y } \\
\text { proteína dietética. }\end{array}$ \\
\hline
\end{tabular}

Tabla 2. Efectos de compuestos alimenticios en el sistema inmune.

\begin{tabular}{|c|c|c|c|c|c|}
\hline Fuente & Compuesto & Tipo de ensayo & Dosis & Efecto observado & $\begin{array}{l}\text { Alimentos en los } \\
\text { que encuentran }\end{array}$ \\
\hline $\begin{array}{l}\text { (Berggren, } \\
\text { Lazou- } \\
\text { Ahrén, } \\
\text { Larsson, } \\
\text { Önning, } \\
\text { 2011) }\end{array}$ & $\begin{array}{l}\text { Probióticos } \\
\text { L. Plantarum } \\
\text { HEALO y L. } \\
\text { paracasei } \\
8700\end{array}$ & $\begin{array}{l}\text { Estudio aleatorizado, } \\
\text { doble ciego y } \\
\text { placebo en } 272 \\
\text { adultos sanos. }\end{array}$ & $\begin{array}{l}\text { Suplementación } \\
\text { diaria con } 10^{9} \\
\text { UFC* }\end{array}$ & $\begin{array}{l}\text { Reducen el riesgo de contraer } \\
\text { infecciones virales de personas } \\
\text { sanas, así como también reduce los } \\
\text { episodios y número de días con } \\
\text { síntomas. }\end{array}$ & $\begin{array}{c}\text { Bebidas lácteas con } \\
\text { probióticos, yogur, } \\
\text { chucrut, kombucha, } \\
\text { suplementos }\end{array}$ \\
\hline $\begin{array}{l}\text { (Goncalves- } \\
\text { Mendes et } \\
\text { al., } 2019\end{array}$ & Vitamina D & $\begin{array}{c}\text { Ensayo aleatorizado, } \\
\text { doble ciego, } \\
\text { controlado con } \\
\text { placebo en adultos } \\
\text { de más de } 65 \text { años. } \\
\end{array}$ & $\begin{array}{c}600.000 \mathrm{UI} \\
\text { diarias* }\end{array}$ & $\begin{array}{l}\text { Promueve un nivel plasmático más } \\
\text { alto de TGFß en respuesta a la } \\
\text { vacunación contra la influenza sin } \\
\text { mejorar la producción de anticuerpos. }\end{array}$ & $\begin{array}{l}\text { Salmón, Trucha, } \\
\text { Sardina }\end{array}$ \\
\hline $\begin{array}{l}\text { (Hornsby et } \\
\text { al., 2018) }\end{array}$ & Vitamina D & $\begin{array}{c}\text { Estudio con el } \\
\text { objetivo de } \\
\text { investigar si la } \\
\text { vitamina C ayuda a } \\
\text { la inmunidad } \\
\text { neonatal en la } \\
\text { suplementación } \\
\text { materna. } \\
\end{array}$ & $\begin{array}{c}4400 \mathrm{UI} / \mathrm{d} \text { en } 26 \\
\text { cordones } \\
\text { umbilicales y } \\
400 \text { UI/d en } 25 \\
\text { cordones } \\
\text { umbilicales. }\end{array}$ & $\begin{array}{c}\text { La exposición a la vitamina D durante } \\
\text { el desarrollo fetal influye en el } \\
\text { sistema inmunológico del recién } \\
\text { nacido, que puede contribuir a la } \\
\text { protección contra los resultados } \\
\text { relacionados con el asma, incluidos } \\
\text { los infecciosos, en los primeros años } \\
\text { de vida. } \\
\end{array}$ & Suplementación \\
\hline $\begin{array}{l}\text { (Hemila, } \\
\text { 2016) }\end{array}$ & Vitamina E & $\begin{array}{c}\text { Ensayo controlado } \\
\text { aleatorio en hombres } \\
\text { fumadores de } 50 \text { a } \\
69 \text { años } \\
\end{array}$ & $50 \mathrm{mg} / \mathrm{dl}$ & $\begin{array}{c}\text { La vitamina E en complemento con el } \\
\text { ejercicio redujo la incidencia de } \\
\text { neumonía en un } 69 \% \text {. }\end{array}$ & $\begin{array}{l}\text { Aceites y } \\
\text { almendras. }\end{array}$ \\
\hline $\begin{array}{l}\text { (Szodoray } \\
\text { et al. 2020) }\end{array}$ & Vitamina A & $\begin{array}{l}\text { Ensayo aleatorizado } \\
\text { doble ciego y } \\
\text { placebo en } 25 \\
\text { pacientes con } \\
\text { Síndrome Sjören } \\
\text { primario (Ssj) y } 15 \\
\text { personas sanas. } \\
\end{array}$ & $\begin{array}{c}\text { Pacientes con } \\
\text { Ssj con } 2 \mu \mathrm{mol} / \text {, } \\
2.5 \mu \mathrm{mol} /, \\
\text { pacientes sanos }\end{array}$ & $\begin{array}{l}\text { Correlación positiva de células NK, } \\
\text { Th17, lo que confirma que la vitamina } \\
\text { A es un regulador de procesos } \\
\text { inmunitarios. }\end{array}$ & $\begin{array}{l}\text { Zanahoria, Hígado y } \\
\text { espinacas. }\end{array}$ \\
\hline $\begin{array}{l}\text { (Waqas- } \\
\text { Khan, } \\
\text { Parikh, } \\
\text { Megala y } \\
\text { Predeteanu } \\
\text {, 2020) } \\
\end{array}$ & Vitamina C & $\begin{array}{c}\text { Estudio con el } \\
\text { objetivo de } \\
\text { investigar si la } \\
\text { vitamina C ayuda a } \\
\text { la recuperación de } \\
\text { COVID-19 } \\
\end{array}$ & $\begin{array}{l}11 \mathrm{~g} \text { por } 24 \mathrm{hrs} \\
\text { por punción } \\
\text { intravenosa } \\
\text { continua }\end{array}$ & $\begin{array}{l}\text { Hemodinámicamente comenzó a } \\
\text { mejorar, la radiografía de tórax } \\
\text { mostró una mejoría en la neumonía, } \\
\text { saturación de oxígeno } 92 \%\end{array}$ & $\begin{array}{l}\text { Frutas cítricas: } \\
\text { Limón, Naranja, } \\
\text { Kiwi. }\end{array}$ \\
\hline $\begin{array}{l}\text { (Kurihara } \\
\text { et al., } \\
\text { 2010) }\end{array}$ & L-teanina & $\begin{array}{c}\text { Ensayo comparativo } \\
\text { aleatorizado, doble } \\
\text { ciego y de grupos } \\
\text { paralelos en } 176 \\
\text { adultos. } \\
\end{array}$ & 700 mg/día & $\begin{array}{l}\text { Suprimió significativamente la } \\
\text { incidencia del resfriado común en } \\
\text { comparación con el grupo placebo. }\end{array}$ & Suplementación \\
\hline
\end{tabular}

* UFC: unidades formadoras de colonias; * "Ul: Unidad internacional; * $\mu$ l: Microlitro. 


\section{Artículo de Revisión}

\section{Discusión}

COVID-19 y su impacto en la salud emocional El COVID-19 es la enfermedad transmitida por el coronavirus SARS-CoV-2, la estructura es esférica y su genoma está constituido por RNA de cadena sencilla. Tiene una envoltura lipídica con tres proteínas ancladas en ellas: E (envoltura), M (membrana) y S (spike), que le da la apariencia de corona, además su tiempo de incubación promedio es de 5 a 14 días (Díaz-Castrillón y Toro-Montoya, 2020). A su vez, la infección ocasionada por el SARS-CoV-2 se asocia con problemas en vías respiratorias que van desde síntomas leves a severos que pueden desencadenar en la muerte (Butlera y Barrientos, 2020; Shakoor et al., 2021).

Por otro lado, se ha demostrado que durante la cuarentena los síntomas psicológicos como el estrés, ansiedad y depresión han aumentado y se asocian con el tiempo extendido de confinamiento, así también con las afectaciones directas que ocasiona el SARSCoV-2 al sistema nervioso central por su potencial capacidad neuro-invasiva que pudieran ocasionar alteraciones en la salud emocional de personas que padecen o han padecido esta enfermedad (Speth et al., 2020; Van-Rheenen et al., 2020; Dubey et al., 2020). El estrés desencadena varios cambios fisiológicos en el sistema nervioso central, órganos y tejidos periféricos entre ellos el incremento de hormonas como el cortisol que produce efectos negativos sobre el organismo (Jaatinen et al., 2014), además el estrés disminuye las defensas del cuerpo dependiendo de la diversidad de factores como la duración, la reacción o la percepción del individuo a la condición estresante afectando además la conducta, capacidad de concentración y de memorización (Naranjo-Pereira, 2009).

Referente a los datos de estrés y ansiedad, China es uno de los países con cifras más altas, el 25\% de la población general ha presentado niveles moderados y severos de ansiedad o síntomas relacionados al estrés en respuesta a la pandemia; asimismo, se ha estudiado el impacto en población estadounidense que previo a la pandemia ya presentaba problemas mentales; en dicha población de estudio se vieron acentuados éstos y otros desórdenes emocionales como xenofobia, miedo a la contaminación, entre otros (Asmundson et al., Ingram, Maciejewski y Hand, 2020). En este sentido, otro estudio realizado en China, evaluó el impacto psicológico de 1,210 personas, y se encontró que el $28.8 \%$ presentó síntomas de ansiedad de moderados a graves, el $16.5 \%$ síntomas depresivos igualmente de moderados a graves y el $8.1 \%$ niveles de estrés elevados (Wang et al., 2020).

Por su parte, en un estudio de España realizado por Pérez y cols., se analizó el estado de ánimo de 1,014 adultos mostrando una correlación directa entre el COVID-19 y los estados de tristeza-depresión, ansiedad y enojo representando el $51 \%$ de la población estudiada (Pérez-Fuentes, Molero-Jurado, Martos-Martínez y Gázquez-Linares, 2020), mientras que otro estudio realizado en Croacia en 3,027 adultos (70\% mujeres) donde se investigó el efecto del confinamiento por COVID-19 en los cambios de humor, se demostró que tanto hombres como mujeres se sintieron asustados $(\mathrm{P}<0,001)$, desanimados $(\mathrm{P}<0,001)$ y tristes $(\mathrm{P}<0,001)$ con la situación actual (Đogaš et al., 2020).

Es indiscutible que el confinamiento por COVID-19 representa una situación estresante para las personas volviéndolas más vulnerables a experimentar sensación de miedo, tristeza, enojo, así como ansiedad o depresión que, de acuerdo con NaranjoPereira son las manifestaciones psicológicas más peligrosas que pueden disminuir significativamente la calidad de vida de quienes lo padecen.

Si bien son más los estudios que refieren y evalúan el impacto psicológico de estrés por el confinamiento, es importante resaltar que la ansiedad y depresión pueden presentarse en personas positivas a COVID-19 por el efecto neuro-invasivo observado como manifestaciones de agitación, confusión, convulsión y posible daño neuronal en el tracto corticoespinal; no obstante, ningún estudio refiere si esta condición emocional persiste después de haber cesado la infección.

Por lo tanto, es de suma importancia proponer un cambio de hábitos en la sociedad que ayude a atenuar los efectos psicológicos de estrés, ansiedad y depresión; uno de ellos es a través del consumo de alimentos mínimamente procesados que contengan componentes bioactivos que muestran beneficios en el estado de ánimo y por ende en el sistema nervioso para modular esta situación de tensión emocional (Yoto, Motoki, Murao y Yokogoshi, 2012). 
Componentes bioactivos, su relación con emociones y sistema inmune

Como medidas de prevención para frenar los contagios de COVID-19, se estableció un confinamiento domiciliario, trayendo como consecuencias cambios en los hábitos alimenticios y comportamientos cotidianos (distanciamiento físico y autoaislamiento) sin olvidar que los contagios incrementaron, por lo que el estrés aumentó de manera significativa. El estrés se asocia con hábitos de consumo poco saludables como comer en exceso y con un mayor riesgo de desarrollar enfermedades que se ha reportado que aumenta el riesgo de complicaciones severas por COVID-19 (Dubey et al., 2020; Speth et al., 2020; Van-Rheenen et al., 2020; Đogaš et al., 2020). Por su parte, diversos autores han descrito que componentes bioactivos presentes en los alimentos como el triptófano, Lteanina, antioxidantes y los probióticos benefician el estado de ánimo cuando se incluyen de manera regular en la dieta diaria.

A continuación, se describen los principales componentes bioactivos reportados que tienen un efecto positivo sobre el estado de ánimo y/o sobre el sistema inmunológico.

\section{Triptófano}

El triptófano (TRP) es un aminoácido esencial mayormente empleado en la síntesis de proteínas; también es precursor de la síntesis de serotonina a nivel intestinal, cerebral y sistema nervioso central. A nivel intestinal, la serotonina modula el peristaltismo y produce una respuesta inflamatoria. Por otro lado, la disminución aguda de TRP en la dieta provoca niveles bajos de serotonina a nivel cerebral produciendo clara dificultad en el control del estado de ánimo en personas sanas, pero con predisposición genética a presentar depresión, no obstante, en pacientes con depresión esta disminución puede derivar en un cuadro depresivo agudo severo (Jenkins, Nguyen, Polglaze y Bertrand, 2016). Asimismo, aproximadamente un $4 \%$ del triptófano ingerido es empleado por el microbiota intestinal que lo transforma en diversos metabolitos con efectos antiinflamatorios. Está bien documentado que altos niveles de estrés emocional o fisiológico pueden alterar la composición de la microbiota intestinal y con ello el metabolismo del TRP que puede inducir un incremento de cortisol y permeabilidad intestinal que finalmente conlleva a una respuesta inmune disminuida (Gao et al., 2018). Un estudio realizado en 2015 evaluó los efectos del TRP dietético en 25 sujetos jóvenes sobre el estado de ánimo positivo (alegría, entusiasmo, etc.) y estado de ánimo negativo (tristeza, ira, miedo, etc.); así como en trastornos de ansiedad y depresión. Se administró una dieta baja en TRP $(<5 \mathrm{mg} / \mathrm{kg}$ de peso corporal/día) y una dieta alta en TRP ( $>10 \mathrm{mg} / \mathrm{kg}$ de peso corporal/día) durante 4 días, ofreciendo alimentos con TRP (queso mozzarella, productos de soya, semillas de calabaza y claras de huevo). Se utilizó la escala de ansiedad de autoevaluación de Zung para evaluar y cuantificar la ansiedad de los participantes en las últimas 24 horas, el estado de ánimo se midió utilizando el PANAS (Programa de Afecto Positivo y Afecto Negativo) y se evaluó la tendencia hacia un estado depresivo empleando la escala de depresión SDS de Zung. Como resultado, se encontró una mejoría en el estado de ansiedad y menor tendencia a la depresión en los participantes que tuvieron una dieta alta en TRP, asimismo, los resultados del test PANAS sobre el estado de irritabilidad demostraron puntuaciones positivas más altas cuando se consumía una dieta alta en TRP (Lindseth, Helland y Caspers, 2015). A su vez, Bravo y cols. concuerdan con lo anterior, en cuyo estudio se muestra una respuesta positiva en el estado de ánimo tras la ingesta de cereales enriquecidos con TRP en 35 adultos de mediana edad y ancianos voluntarios durante 3 semanas; en la primer semana consumieron cereales $(22.5 \mathrm{mg}$ de TRP en $30 \mathrm{~g}$ de cereales por dosis) en el desayuno y la cena, en la segunda semana consumieron cereales con dosis más alta de TRP (60 $\mathrm{mg}$ de TRP en $30 \mathrm{~g}$ de cereales por dosis) en el desayuno y cena y en la tercer semana dieta habitual. Se monitorearon por tres semanas y analizaron muestras de orina para medir la serotonina total. Como resultado encontraron que los cereales con dosis más altas de TRP aumentaron los niveles de serotonina en orina y mejoraron los síntomas de depresión de acuerdo con el test de Beck (Bravo et al., 2013). A través de una adecuada ingesta de TRP es posible mantener el equilibrio entre la tolerancia inmunológica intestinal y el mantenimiento de la microbiota (Gao et al., 2018) como vía reguladora de las respuestas inflamatorias (Marsland, 2016).

Estos estudios, aunque concuerdan con el rol predominante del triptófano y sus metabolitos en la homeostasis de la microbiota y el sistema inmune, así 
como su papel en el estado de ánimo, no todos resaltan el rol que juega la vitamina B6 en el transporte del triptófano desde el torrente sanguíneo al cerebro para posteriormente ser convertido en serotonina. Por otro lado, las muestras de sujetos de estudio son pequeñas y no se incluyen personas con un diagnóstico definido de ansiedad o depresión, ni tampoco con antecedentes familiares identificados; es importante resaltar que, aunque las dosis y tiempos de tratamientos fueron diferentes en cada estudio, son claros los efectos positivos del TRP en el estado de ánimo tanto en personas jóvenes como adultos mayores consumiendo alimentos enriquecidos en este aminoácido.

\section{L-Teanina}

La L-Teanina es otro aminoácido que se encuentra de forma natural en el té verde $y$ otras plantas (Sakamoto, Pereira, Bueno y Santos, 2019), su función es bloquear la unión del ácido L-glutámico a los receptores de glutamato en el cerebro e inhibir la excitación de las neuronas corticales mostrando efectos antiestrés. En un estudio en 2012, se investigó los efectos de la L-teanina sobre el estrés psicológico en 14 personas sanas durante 7 días, sometiendo a cada uno a tres sesiones por separado administrando por vía oral $200 \mathrm{mg}$ de L-teanina más placebo (Dextrina), $100 \mathrm{mg}$ de cafeína más placebo o solo placebo en conjunto con $250 \mathrm{~mL}$ de agua. Se les midió la presión arterial y se expusieron a 3 sesiones de carga de estrés, realizando en cada una de ellas tareas mentales y tareas de estrés físico después de cada tratamiento. La evaluación fue con el Profile of Mood States (POMS) para medir el estado de ánimo de tensión y ansiedad; los resultados de las puntuaciones de POMS indicaron que la Lteanina muestra efecto anti estrés ya que disminuyó significativamente la presión arterial a través de inhibir la excitación de las neuronas corticales y la respuesta de activación nerviosa simpática en presencia de estrés, además, la ingesta de L-teanina mejoró el estado de ánimo de los participantes al reducir la tensión y la ansiedad causadas por el estrés psicológico en comparación con el placebo (Yoto, Motoki, Murao y Yokogoshi, 2012).En comparación, otro estudio evaluó el efecto de una bebida nutritiva a base L-teanina sobre el estado de ánimo y un factor de estrés cognitivo. La investigación se realizó en 34 adultos sanos de entre 18 y 40 años. Se evaluó el rendimiento cognitivo y la actividad oscilatoria alfa del estado de reposo (potencial neurofisiológico) con la magneto encefalografía (MEG). El estado de estrés, como el estado de ánimo y fatiga fueron evaluados antes y después de esta herramienta empleando test de Índice de ansiedad STAI-S (State-Trait Anxiety Index). El resultado apoya los efectos antiestrés de L-teanina, ya que el consumo de $200 \mathrm{mg}$ de la bebida nutritiva, con dosis pequeñas de $1 \mathrm{mg}$ de fosfatidilserina, 25 $\mathrm{mg}$ de alfa glicerilfosforilcolina y $10 \mathrm{mg}$ de manzanilla como vehículos, redujo la respuesta subjetiva al estrés significativamente después de una hora de ingerir la dosis y redujo el cortisol tres horas después de ingerir la dosis (White et al., 2016). Por otro lado, algunos autores sugieren que la L-teanina tiene efecto en la respuesta inmune humoral asociada con la producción de anticuerpos; en un ensayo aleatorizado doble ciego de 5 semanas participaron 176 hombres sanos, a los que se les administró 2 tabletas de cistina y teanina por vía oral después del desayuno y la cena, cada tableta con 175 mg de L- cistina más $70 \mathrm{mg}$ de L -teanina para tratar los síntomas de la resfriado común, obteniendo una reducción significativa de incidencia de resfriado y síntoma de fiebre (Kurihara et al., 2010). A pesar de estos hallazgos, es importante realizar más estudios para concretar que L-teanina tiene un efecto en el estado inmune, ya que en esta revisión no se encontraron más estudios para determinar que su uso de forma individual es efectivo para reforzar la respuesta inmune.

\section{Antioxidantes}

Los antioxidantes son un grupo de compuestos que se encuentran en los alimentos, dentro de sus principales beneficios se encuentra prevenir los efectos de radicales libres en funciones fisiológicas en humanos.

Un ensayo clínico aleatorizado a doble ciego en 20 adultos sanos midió el efecto de los polifenoles en estado de ánimo subjetivo (calma, alerta, relajado, tenso); los compuestos fenólicos que se encuentran en las bayas de uvas se han relacionado con aumento de la función cerebral y estado cognitivo. En la evaluación del estado de ánimo subjetivo se utilizaron las Escalas de Bond-Lader; a su vez, el contenido fenólico se determinó mediante el método Folin-Ciocalteu. Los resultados sugieren que el consumo de $200 \mathrm{~mL}$ de jugo de uva morada del cual $65 \%$ de contenido es de uva Concord, puede mejorar el estado de ánimo en adultos jóvenes. Los 
participantes estaban más tranquilos después de consumir el jugo de uva en comparación al grupo placebo, además se tuvo resultados significativos en el tiempo de atención compuesta $(\mathrm{p}=0,047)$ y aumento en las calificaciones de calma $(p=0,047)$ (Haskell-Ramsay, Stuart, Okello y Watson, 2017). Un estudio realizado por Khalid en 2017 evaluó el cambio del estado de ánimo en 21 adultos sanos a los que se les entregó una bebida con un total de $253 \mathrm{mg}$ de antocianinas y una bebida placebo de vitamina $\mathrm{C}$ con fructosa. Los participantes asistieron al laboratorio durante un mínimo de tres a siete días. El resultado mostró un efecto agudo tras el consumo de flavonoides de arándanos sobre el afecto positivo y ningún efecto sobre el afecto negativo en adultos jóvenes sanos, esto se debe a que los flavonoides se han asociado con la función ejecutiva (FE), una FE deteriorada mantiene un estado de depresión y estado de ánimo bajo y una FE mejorada reduce los procesos cognitivos depresivos (Khalid et al., 2017). Consistente con este resultado Haskell en el 2017 evaluó la mejoría del estado de ánimo en adultos jóvenes sanos tras la suplementación con jugo de uva morada. Se realizó con 20 participantes, se administró el tratamiento $(200 \mathrm{~mL}$ de jugo de uva morada y $30 \mathrm{~mL}$ de cordial sabor grosella negra) y el placebo $(200 \mathrm{~mL}$ de jugo de uva blanca, $10 \mathrm{~mL}$ de cordial sabor grosella negra y $20 \mathrm{~mL}$ de agua fría). Como resultado obtuvieron que el jugo de uva morada aumentó las calificaciones de calma en los pacientes, mejorando su estado mental ante cualquier alteración causado por ansiedad o depresión (Haskell et al., 2017).

En la tabla 1 se muestran los compuestos bioactivos en los que se observaron efectos positivos sobre el estado de ánimo.

\section{Vitamina $C$}

La vitamina $\mathrm{C}$ es un antioxidante que protege del daño oxidativo generado durante el metabolismo celular o por exposición a toxinas y contaminantes externos, a la vez tiene un efecto en el sistema inmune innato ya que estimula la migración de neutrófilos al sitio de la infección; su acumulación en las células de neutrófilos puede mejorar la quimiotaxis, la fagocitosis y la eliminación microbiana (Carr y Maggini, 2017).

Waqas-Khan y cols., realizaron un estudio en pacientes de 74 años con el diagnóstico de COVID-
19 con síntomas de fiebre baja, tos seca y disnea. Durante la exploración presentaron los siguientes signos vitales: temperatura de $37.7^{\circ}$, presión arterial de 121/82, pulso de 87 latidos por minuto (lpm), frecuencia respiratoria de 16 y saturación de oxígeno del $87 \%$. El séptimo día intrahospitalario se inició con una dosis alta de Vitamina C; $11 \mathrm{~g}$ por $24 \mathrm{hrs}$ por punción intravenosa continua hasta el día 16 intrahospitalario. Como resultado, el estado clínico mejoró lentamente. El soporte de noradrenalina se suspendió al día noveno, la radiografía de tórax mostró una mejoría en la neumonía, su estado respiratorio continuó mejorando con una saturación de oxígeno de $92 \%$ y sin necesidad de ventilación mecánica, por lo que se le atribuye la mejora a la administración de Vitamina $\mathrm{C}$ en conjunto con los medicamentos utilizados (Waqas-Khan, Parikh, Megala y Predeteanu, 2020).

En nuestra revisión no se encontraron estudios sobre su efectividad de la vitamina $\mathrm{C}$ en el estado de ánimo y en la tabla 2 se muestran los componentes que mostraron efectos positivos sobre el sistema inmune.

\section{Probióticos}

Los probióticos son microorganismos vivos que mejoran el balance de la microbiota intestinal; sus efectos benéficos sobre la salud humana los ejercen a través del eje cerebro-intestino cuya comunicación se genera a través del nervio vago, vía neuroendocrina y rutas de respuesta inmune (RI); de esta forma la inflamación intestinal puede reflejar una subsecuente neuroinflamación. Recientemente, se están estudiando múltiples cepas probióticas considerándolas como tratamiento adyuvante en cuadros depresivos. El estudio de Steenbergen L y cols. en el 2015, realizado en 40 pacientes durante 4 semanas, empleó una suplementación con probióticos de múltiples especies descritos en la tabla 1, como estrategia adyuvante para mejorar la depresión. Esto se evaluó antes y después con el Índice de Sensibilidad a la Depresión de Leiden (LEIDS-r). Se mostró una reducción en la depresión y los pensamientos agresivos asociados con el estado de ánimo triste como angustia, pérdida de motivación $\mathrm{y}$ expectativas sobre el futuro, etc. (Steenbergen et al., 2015).

Estos resultados son consistentes con lo reportado por Akkasheh y cols. en 2016, en 40 pacientes durante 8 semanas tratados con probióticos de las 
cepas descritas en la tabla 1 . Los resultados fueron una disminución significativa de los síntomas de depresión según las puntuaciones totales del Inventario de Depresión de Beck en comparación con el placebo (almidón) (Steenbergen et al., 2015; Akkasheh et al, 2016); no obstante, se debe investigar sobre los fundamentos neurobiológicos de los efectos observados en los estudios anteriores. Además de presentar efectos positivos sobre el estado de ánimo, otros estudios muestran que los probióticos tienen efectos inmunorreguladores mediante las células inmunitarias sistémicas y mucosas, así como las células epiteliales intestinales activando la inmunidad innata para preparar las respuestas inmunitarias adaptativas, teniendo un potencial terapéutico para enfermedades relacionadas con la RI, como alergia, infección viral y potenciación de las respuestas de vacunación (Yan y Polk, 2011). En este sentido, Berggren, A y cols., en el 2011 realizaron un estudio en donde se suplementó diariamente con 109 UFC (Unidades Formadoras de Colonias) a 272 personas sanas con cepas probióticas L. Plantarum HEALO y L. paracasei 8700: 2, durante 12 semanas. El resultado fue que la incidencia de adquirir uno o más episodios de infecciones virales se redujo de $67 \%$ en el grupo control a $55 \%$ en el grupo de probióticos ( $\mathrm{p}<0.05)$, el número de días con síntomas se redujo significativamente $(\mathrm{p}<0.05)$ de 8.6 días en el grupo de control a 6.2 días en el grupo de probióticos, durante el período de 12 semanas (Berggren, LazouAhrén, Larsson, Önning, 2011), pudiendo concluir que las cepas probióticas de Lactobacillus, Bifidobacterium son eficaces como tratamiento adyuvante para mejorar o prevenir la depresión y mejorar el sistema inmune.

\section{Vitamina D}

La Vitamina D (VD) es un precedente de la hormona esteroidea que es esencial para el organismo humano. Uno de sus orígenes es endógeno, cuando se expone a la radiación ultravioleta $B$ (UVB) en la epidermis de la piel en donde se transforma en colecalciferol, el cual se hidroxila en el hígado para formar 25hidroxivitamina $\mathrm{D}$, que se transforma en la hormona 1,25-hidroxivitamina la cual termina en el riñón como Calcitriol (Shakoor et al., 2021); o exógeno por medio de la ingestión de verduras (vitamina D2, ergocalciferol) o de origen animal (colecalciferol) (Bizzaro, Antico y Fortunato, 2017). En 2019 se realizó un ensayo aleatorizado con placebo en donde se investigó si la suplementación en personas mayores de 65 años mejoraría la RI para evitar el riesgo de contagio por influenza; se dividieron los voluntarios en dos grupos: el primero con un nivel sérico de $\mathrm{VD} \geq 30 \mathrm{ng} / \mathrm{mL}$ y el segundo con un nivel $<30 \mathrm{ng} / \mathrm{mL}$. Fueron asignadas al azar a uno de dos grupos en donde uno se suplementó durante 3 meses con una dosis de 100,000 UI durante 15 días, seguido de una vacuna de la influenza; el otro grupo recibió un placebo en un lapso de 3 meses, seguido de una vacuna contra la influenza. Se concluyó que la suplementación con VD en respuesta a la vacunación dirige la polarización de los linfocitos hacia una RI tolerogénica, así como impulsa un mayor nivel plasmático de TGFß (Factor de crecimiento transformador-beta), sin embargo, no mejora la reproducción de anticuerpos (Goncalves-Mendez, 2019).

Así mismo, en 2018 se realizó un ensayo clínico aleatorizado doble ciego y controlado con placebo en 51 mujeres embarazadas con el objetivo de investigar el efecto sobre la inmunidad neonatal de la suplementación materna mediante el subconjunto de muestras de sangre del cordón umbilical, las pacientes estaban entre la 10-18 semanas de embarazo para recibir suplementos de VD que se dividieron en dos grupos: dosis altas $4400 \mathrm{UI} / \mathrm{d}$ de VD $(n=25)$ y dosis bajas de 440 UI/d de VD $(n=26)$ Como resultado del estudio el grupo de dosis altas tuvo una respuesta mejorada de citocinas proinflamatorias, brindando un beneficio en el desarrollo en el sistema inmunológico del recién nacido, así mismo se concluyó que la suplementación de la VD durante el desarrollo fetal tiene un impacto en el sistema inmunológico, ya que puede contribuir a la protección contra el asma, e infecciones de vías respiratorias (Hornsby, et al., 2018).

Por otro lado, se ha observado que la VD también tiene un efecto en las emociones, al ser una hormona neuroesteroidea única. Por medio de la regulación de la síntesis de neurotransmisores, mejora el factor de crecimiento nervioso, así como el estado de ánimo. Esta vitamina está involucrada en diversos procesos cerebrales, tales como neuroinmunomodulación, regulación de factores neurotróficos, neuroprotección, neuroplasticidad y desarrollo cerebral (Anglin, Samaan, Walter y McDonald, 2013). En el año 2017 se realizó un ensayo doble ciego controlado con placebo, se trataron 33 
pacientes de 18-70 años con depresión bipolar y deficiencia de VD. Los sujetos fueron divididos en dos grupos: uno con suplementación de 5000 UI de VD y otro placebo. Para medir la depresión bipolar se midió con puntuación MADRS (Montgomery Asberg Depression Rating Scale), YMRS (Young Mania Rating Scale) y HAM-A (Hamilton Depression Rating Scale). Los principales resultados fueron que la puntuación MADRS disminuyó significativamente en el grupo placebo de igual forma en el grupo con VD lo que significa que ambos tuvieron una disminución significativa de los síntomas de depresión (Marsh, Penny y Rothschild, 2017). Por lo que se requieren más estudios para esclarecer el papel de la vitamina D sobre la depresión y ansiedad.

\section{Vitamina $E$}

La Vitamina $\mathrm{E}$ es un antioxidante compuesto por cuatro tocoferoles y cuatro tocotrienoles (Khadangi y Azzi, 2018). En 2016 se realizó un ensayo controlado aleatorio, donde su principal objetivo era suplementar vitamina E para analizar la disminución de incidencia de neumonía en hombres fumadores de 50 a 69 años con la característica de que fumaran más de 5 cigarrillos al día, se les suplementó con 50 $\mathrm{mg} / \mathrm{dL}$ de vitamina $\mathrm{E}$ durante 5-8 años, 2216 pacientes fueron asignados al azar a cuatro grupos: placebo, Alfa-tocoferol, Betacaroteno + Alfatocoferol, Betacaroteno. Los resultados comprobaron que el grupo Alfa-tocoferol (Vitamina E), en complemento con el ejercicio en tiempo libre redujo la incidencia de neumonía en un 69\% (Hemila, 2016). Al encontrar solo un artículo sobre el efecto de Vitamina E sobre el sistema inmunológico por lo tanto se requieren más estudios que ayuden a esclarecer la participación de la vitamina E sobre el sistema inmune.

\section{Vitamina A}

La Vitamina A es un micronutriente que tiene un papel significativo en procesos fisiológicos, como lo es la visión, la respuesta inmunitaria, la diferenciación y proliferación celular (Debelo, Novotny y Ferruzzi, 2017). Se han hecho diversos estudios relacionando el sistema inmunológico y la ingesta de vitamina A. En 2010, se realizó un estudio para investigar el papel inmunomodulador de la vitamina A en 25 pacientes con Síndrome Sjören primario (Ssj) y 15 personas sanas. Se encontró que las concentraciones de vitamina $\mathrm{A}$ en el plasma disminuyeron significativamente en personas con $\mathrm{Ssj}$, ya que los pacientes sanos conservaban $2.5 \mu$ mol/L, comparado con los pacientes con $\mathrm{Ssj}$ con $2 \mu$ mol/L, demostrando la correlación positiva que existe entre los porcentajes de células NK, Th17 y los niveles de vitamina $\mathrm{A}$, ya que la producción de citocinas, células N, Th17 están asociadas comúnmente con respuestas inmunitarias. Esto confirma que la vitamina $\mathrm{A}$ es un regulador de procesos inmunitarios, por lo que su disminución favorece al desarrollo de infecciones virales o bien la presencia de manifestaciones más graves (Szodoray et al., 2010).

En la presente revisión no se encontraron estudios sobre su efectividad de la vitamina A en el estado de ánimo.

A nuestro conocimiento, esta revisión es la primera que se enfoca en identificar componentes bioactivos que influyen positivamente tanto en la respuesta inmune como en el estado de ánimo, principales aspectos de salud que se han visto afectados durante la actual emergencia sanitaria por COVID-19; no obstante, este trabajo presenta ciertas limitaciones ya que los estudios seleccionados miden la respuesta en diversos estados de ánimo como tristeza, ira, miedo, tensión, estrés incluyendo ansiedad y depresión; la diversidad de estados de ánimo dificulta la interpretación de los resultados por el empleo de mayor variedad de herramientas metodológicas en las poblaciones estudiadas. Además, en los estudios incluidos se emplearon preparaciones nutracéuticas con dosis establecidas en las poblaciones estudiadas, por lo que no es posible hacer recomendaciones dietéticas precisas a nuestra población, sólo nos limitamos a promover el consumo de alimentos que contengan este tipo de componentes benéficos, haciendo énfasis en los que muestran doble efecto. Aun así, tales limitantes nos muestran la necesidad de realizar estudios que se enfoquen en evaluar componentes bioactivos que presenten más de un efecto positivo en la salud.

\section{Conclusiones}

El confinamiento por la pandemia de COVID-19 ha generado tensión emocional en la población, incrementando los estados de ansiedad, estrés y/o depresión que pueden tener una influencia negativa sobre el sistema inmunológico, confiriendo mayor 
vulnerabilidad a contraer infecciones. La presente revisión tuvo como propósito mostrar diferentes compuestos alimenticios que influyen tanto en la mejora del estado de ánimo como en la RI. Los principales compuestos que se destacan por tener un efecto antiestrés que promueve un estado de calma y relajación y disminuyen los síntomas de ansiedad son el triptófano, la L-teanina y polifenoles encontrados en arándanos y uva morada clase Concord. Con base en los diferentes estudios analizados, los probióticos de los géneros Lactobacillus acidophilus, casei, brevis, salvarius; Lactocuccus lactis, Bifidobacterium bifidum y lactis mejoran los estados de ansiedad y depresión. Por su parte, las vitaminas A, D, E y C, así como los probióticos Lactobacillus plantarum y paracasei y la L-teanina muestran un efecto positivo en la respuesta del sistema inmunológico reduciendo el riesgo de infecciones. Asimismo, se observó que los probióticos y la Lteanina son los que destacan por presentar ambos efectos.

Es importante resaltar que, aunque las dosis empleadas de estos componentes, sujetos y tiempos de tratamientos fueron diferentes en cada estudio, son claros los efectos positivos que éstos muestran en el estado de ánimo y respuesta inmune.

Esta revisión pretende servir de motivación en el mejoramiento y adecuación de programas de alimentación y nutrición dirigidos a grupos vulnerables, además de proporcionar información útil que ayude al diseño de nuevas estrategias en nutrición para mejorar la salud mental y nutricional teniendo un mejor pronóstico durante el confinamiento o infección por Covid-19.

Asimismo, se destaca la importancia de investigar los posibles efectos sinérgicos tras el consumo de una combinación de varios de este tipo de compuestos.

\section{Bibliografía}

Akkasheh, G., Kashani-Poor, Z., Tajabadi-Ebrahimi, M., Jafari, P., Akbari, H., et al. (2016). Clinical and metabolic response to probiotic administration in patients with major depressive disorder. Nutrition. 32(3), 315-320. doi: 10.1016/j.nut.2015.09.003
Anglin, R., Samaan, Z., Walter, S., \& McDonald, S. (2013). Vitamin D deficiency and depression in adults: Systematic review and meta-analysis. $\mathrm{Br}$ J Psychiatry, 202(2), 100-107. doi:10.1192/bjp.bp.111.106666

Asmundson, G. J., Paluszek, M. M., Landry, C. A., Rachor, G. S., McKay, D., \& Taylor, S. (2020). Do pre-existing anxiety-related and mood disorders differentially impact COVID-19 stress responses and coping? J Anxiety Disord. 74, 102271. doi.org/10.1016/j.jiph.2020.06.021

Berggren, A., Lazou-Ahrén, I., Larsson, N., Önning, G. (2011). Randomised, double-blind and placebo-controlled study using new probiotic lactobacilli for strengthening the body immune defence against viral infections. Eur $J$ Nutr, 50(3), 203-10. doi: 10.1007/s00394-010-0127-6

Bizzaro, G., Antico, A., Fortunato, A., \& Bizzaro, N. (2017). Vitamin D and Autoimmune Diseases: Is Vitamin D Receptor (VDR) Polymorphism the Culprit? Isr Med Assoc J: IMAJ, 19(7), 438-443. PMID: 28786260

Bravo, R., Matito, S., Cubero, J., Paredes, S. D., Franco, L., Rivero, M., Rodríguez, A. B., \& Barriga, C. (2013). Tryptophan-enriched cereal intake improves nocturnal sleep, melatonin, serotonin, and total antioxidant capacity levels and mood in elderly humans. Age (Dordrecht, Netherlands), 35(4), 1277-1285. doi.org/10.1007/s11357-012-9419-5

Butlera, M. J., \& Barrientos, R. M. (2020). The impact of nutrition on COVID-19 susceptibility and long-term consequences. Brain Behav Immun, 87, 53-54. doi.org/10.1016/j.bbi.2020.04.040

Camacho Arroyo, I. (2020). Estado de Animo incide en la salud. Dirección General de Comunicación social.

https://www.dgcs.unam.mx/boletin/bdboletin/20 20_864.html

Carr, A. C., \& Maggini, S. (2017). Vitamin C and Immune Function. Nutrients, 9(11), 1211. doi.org/10.3390/nu9111211 
Debelo, H., Novotny, J. A., \& Ferruzzi, M. G. (2017). Vitamin A. Advances in Nutrition: $A n$ International Review Journal, 8(6), 992-994. doi.org/10.3945/an.116.014720

Díaz-Castrillón, F. J., \& Toro-Montoya, A. I. (2020). SARS-CoV-2/COVID-19: el virus, la enfermedad y la pandemia. Med Lab, 24(3), 183205. doi.org/10.36384/01232576.268

Đogaš, Z., Kalcina, L. L., Dodig, I. P., Demirović, S., Madirazza, K., Valić, M., \& Pecotić, R. (2020). The effect of COVID-19 lockdown on lifestyle and mood in Croatian general population: a crosssectional study. Croat Med J. 61(4), 309. doi: 10.3325 / cmj.2020.61.309

Dubey, S., Biswas, P. Ghosh, R. Chattarjee, S. Dubey, M. J., Chatterjee, S., Lahiri, D., Lavie, C. J. (2020). Psychosocial impact of COVID-19. Diabetes Metab Syndr, 14(5), 779-788. doi: 10.1016/j.dsx.2020.05.035

Gao, J., Xu, K., Liu, H., Liu, G., Bai, M., Peng, C., Li, T., Yin, Y. (2018). Impact of the Gut Microbiota on Intestinal Immunity Mediated by Tryptophan Metabolism. Front Cell Infect Microbiol, 6,8-13. doi: 10.3389/fcimb.2018.00013

Goncalves-Mendes, N., Talvas, J., Dualé, C., Guttmann, A., Corbin, V., et al. (2019). Impact of Vitamin D Supplementation on Influenza Vaccine Response and Immune Functions in Deficient Elderly Persons: A Randomized Placebo-Controlled Trial. Front Immunol, 10; 65. doi.org/10.3389/fimmu.2019.00065

Haskell-Ramsay, C. F., Stuart, R. C., Okello, E. J., \& Watson, A. W. (2017). Cognitive and mood improvements following acute supplementation with purple grape juice in healthy young adults. Eur J Nutr, 56(8), 2621-2631. doi.org/10.1007/s00394-017-1454-7

Hemila, H. (2016). Vitamin E administration may decrease the incidence of pneumonia in elderly males. Clinical Interventions in Aging, Volume 11, 1379-1385. doi.org/10.2147/cia. s114515
Hornsby, E., Pfeffer, P. E., Laranjo, N., Cruikshank, W., Tuzova, M., Litonjua, A. A., Weiss, S. T., Carey, V. J., O’Connor, G., \& Hawrylowicz, C. (2018). Vitamin D supplementation during pregnancy: Effect on the neonatal immune system in a randomized controlled trial. J Allergy Clin Immunol, 141(1), 269-278.e1. doi.org/10.1016/j.jaci.2017.02.039

Infusino, F., Marazzato, M., Mancone, M., Fedele, F., Mastroianni, et al. (2020). Diet Supplementation, Probiotics, and Nutraceuticals in SARS-CoV-2 Infection: A Scoping Review, Nutrients. 12(6), 1718. doi.org/10.3390/nu12061718

Ingram, J., Maciejewski, G., \& Hand, C. J. (2020). Changes in diet, sleep, and physical activity are associated with differences in negative mood during COVID-19 lockdown. Front Psychol. 11, 2328. doi.org/10.3389/fpsyg.2020.588604

Jaatinen, N., Korpela, R., Poussa, T., Turpeinen, A., Mustonen, S., Merilahti, J., Peuhkuri, K., (2014). Effects of daily intake of yoghurt enriched with bioactive components on chronic stress responses: a double-blinded randomized controlled trial. Int J Food Sci Nutr, 65(4), 507514. doi: 10.3109/09637486.2014.880669

Jenkins, T. A., Nguyen, J. C., Polglaze, K. E., \& Bertrand, P. P. (2016). Influence of Tryptophan and Serotonin on Mood and Cognition with a Possible Role of the Gut-Brain Axis. Nutrients, 8(1); 56. doi.org/10.3390/nu8010056

Khadangi, F., \& Azzi, A. (2019). Vitamin E - The Next 100 Years. IUBMB life, 71(4), 411-415. doi.org/10.1002/iub.1990

Khalid, S., Barfoot, K. L., May, G., Lamport, D. J., Reynolds, S. A., \& Williams, C. M. (2017). Effects of Acute Blueberry Flavonoids on Mood in Children and Young Adults. Nutrients, 9(2), 158. doi.org/10.3390/nu9020158

Kurihara, S., Hiraoka, T., Akutsu, M., Sukegawa, E., Bannai, M., \& Shibahara, S. (2010). Effects of (L)-cystine and (L)-theanine supplementation on the common cold: a randomized, double-blind, 
and placebo-controlled trial. $J$ Amino Acids, 2010, 307475. DOI: 10.4061/2010/307475

Lindseth, G., Helland, B. y Caspers, J., (2015). The Effects of Dietary Tryptophan on Affective Disorders. Arch Psychiatr Nurs, 29(2), 102-7. doi: 10.1016/j.apnu.2014.11.008

Marsh, W. K., Penny, J. L., \& Rothschild, A. J. (2017). Vitamin D supplementation in bipolar depression: A double blind placebo controlled trial. J Psychiatr Res, 95, 48-53. doi.org/10.1016/j.jpsychires.2017.07.021

Marsland, B.J. (2016). Regula la inflamación con metabolitos microbianos. Nat. Medicina. 22, 581-583. 10.1038 / nm.4117

Naranjo-Pereira, M. L. (2009). Una revisión teórica sobre el estrés y algunos aspectos relevantes de éste en el ámbito educativo. Rev. Educación: 33 (2), 171-190. DOI 10.15517/REVEDU.V33I2.511

Pérez-Fuentes, M. D. C., Molero Jurado, M. D. M., Martos Martínez, Á., \& Gázquez Linares, J. J. (2020). Threat of COVID-19 and emotional state during quarantine: Positive and negative affect as mediators in a cross-sectional study of the Spanish population. PloS one, 15(6). doi.org/10.1371/journal.pone.0235305

Sakamoto, F., Pereira, R., Bueno, A., Santos, H. (2019). Psychotropic effects of L-theanine and its clinical properties: From the management of anxiety and stress to a potential use in schizophrenia. Pharmacol Res, 147. doi.org/10.1016/j.phrs.2019.104395

Shakoor, H., Feehan, J., Al Dhaheri, A. S., Ali, H. I., Platat, C., Ismail, L. C., Stojanovska, L. (2021). Immune-boosting role of vitamins D, C, E, zinc, selenium and omega- 3 fatty acids: Could they help against COVID-19? Maturitas, 143, 1-9. doi.org/10.1016/j.maturitas.2020.08.003

Speth, M. M., Singer-Cornelius, T., Oberle, M., Gengler, I., Brockmeier, S. J., \& Sedaghat, A. R. (2020). Mood, anxiety and olfactory dysfunction in COVID-19: evidence of central nervous system involvement? The Laryngoscope 130(11). doi.org/10.1002/lary.28964

Steenbergen L, Sellaro R, van Hemert S, Bosch JA, Colzato LS. (2015). A randomized controlled trial to test the effect of multispecies probiotics on cognitive reactivity to sad mood. Brain Behav Immun, $\quad 48 ; \quad$ 258-64. doi.org/10.1016/j.bbi.2015.04.003

Szodoray P, Horvath IF, Papp G, Barath S, Gyimesi E, Csathy L, Kappelmayer J, Sipka S, Duttaroy AK, Nakken B, Zeher M (2010). The immunoregulatory role of vitamins $\mathrm{A}, \mathrm{D}$ and $\mathrm{E}$ in patients with primary Sjogren's syndrome. Rheumatology (Oxford). 49(2); 211-7. doi: 10.1093/rheumatology/kep374

Van-Rheenen, T. E., Meyer, D., Neill, E., Phillipou, A., Tan, E. J., et al. (2020). Mental health status of individuals with a mood-disorder during the COVID-19 pandemic in Australia: Initial results from the COLLATE project. Pharmacol Res. 275; 69-77. doi.org/10.1016/j.jad.2020.06.037

Wang, C., Pan, R., Wan, X., Tan, Y., Xu, L., et al. (2020). Immediate psychological responses and associated factors during the initial stage of the 2019 coronavirus disease (COVID-19) epidemic among the general population in China. Int $J$ Environ Res Public Health, 17(5), 1729. Doi: 10.3390 / ijerph17051729

Waqas Khan, H. M., Parikh, N., Megala, S. M., \& Predeteanu, G. S. (2020). Unusual Early Recovery of a Critical COVID-19 Patient After Administration of Intravenous Vitamin C. Am J Case Rep, 21. doi.org/10.12659/ajcr.925521

White, D. J., de Klerk, S., Woods, W., Gondalia, S., Noonan, C., \& Scholey, A. B. (2016). AntiStress, Behavioural and Magnetoencephalography Effects of an LTheanine-Based Nutrient Drink: A Randomised, Double-Blind, Placebo-Controlled, Crossover Trial. $\quad$ Nutrients, $\quad 8(1), \quad 53$. doi.org/10.3390/nu8010053

Wibawa, T. (2020). COVID-19 vaccine research and development: ethical issues. Tropical Medicine 


\section{RESPYN}

\& International Health, 26(1), 14-19. https://doi.org/10.1111/tmi.13503

Yan, F. y Polk, DB (2011). Probiotics and immune health. Opinión actual en gastroenterología, 27(6), 496-501. doi.org/10.1097/MOG.0b013e32834baa4d

Yoto, A., Motoki, M., Murao, S., Yokogoshi. (2012). Effects of L-theanine or caffeine intake on changes in blood pressure under physical and psychological stresses. J Physiol Anthropol, 31(1), 1-9. doi: 10.1186/1880-6805-31-28 